\title{
Diagnosing acute aortic syndrome: a Canadian clinical practice guideline
}

\author{
Robert Ohle MBBCh MSc, Justin W. Yan MD MSc, Krishan Yadav MD MSc, Alexis Cournoyer MD, \\ David W. Savage MD PhD, Prasad Jetty MD, Rony Atoui MD MSc, Bindu Bittira MD MSc, Brock Wilson MD, \\ Ashish Gupta MD, Niamh Coffey MD, Yvonne Callaway MA MBA, Jeffrey Middaugh MD MSc, \\ Dominique Ansell MD MSc, Fraser Rubens MD, Stephen J. Bignucolo MD, Terena-Marie Scott BSc RN, \\ Sarah Mclsaac MBBCh MEd, Eddy Lang MD MSc
}

Cite as: CMAJ 2020 July 20;192:E832-43. doi: 10.1503/cmaj.200021

CMAJ Podcasts: author interview at https://soundcloud.com/cmajpodcasts/200021

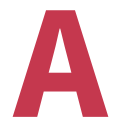

cute aortic syndrome (AAS) is a life-threatening emergency, accounting for $1 / 2000$ presentations of acute chest or back pain to the emergency department. ${ }^{1}$ It is a clinical spectrum of diagnoses including aortic dissection, intramural hematoma and penetrating atherosclerotic ulcer at any location along the aorta. ${ }^{1}$ The incidence of AAS is about 3 per 100000 persons. ${ }^{2,3}$ Many physicians do not consider AAS in their initial differential diagnosis, which is in part why $25 \%$ of patients with AAS are not diagnosed with the condition until 24 hours after presenting to the emergency department. ${ }^{4}$

Prognosis is most favourable when patients are treated early, while they are clinically stable. Mortality follows a linear increase with diagnostic delay and can be as high as $2 \%$ per hour of delay. ${ }^{5}$ The misdiagnosis rate during the initial emergency department visit for AAS (i.e., patient admitted for an alternative diagnosis and later diagnosed as AAS; discharged from the emergency department and presenting again with a diagnosis of AAS; or diagnosed on postmortem examination) is estimated to be as high as 38\%.4, ${ }^{4,-16}$

Patients with suspected AAS are typically investigated with electrocardiogram (ECG)-gated contrast-enhanced computed tomography (CT). ${ }^{2}$ Current use of this investigation in patients with a clinical suspicion for AAS is inefficient. ${ }^{17,18}$ The unnecessary use of CT leads to a direct increase in health care costs but also an increase in contrast-associated complications (e.g., allergic reactions), increased length of emergency department stay or incidental findings requiring further follow-up, additional imaging and increased stress or anxiety for the patient. ${ }^{17}$ Use of CT in a low-prevalence population can result in an increase in false-positives, which can lead to further testing, unnecessary transfer and even surgical intervention. ${ }^{19}$

There are 2 high-quality guidelines related to the diagnosis of AAS, from the American Heart Association (2010) and the European

\section{KEY POINTS}

- This clinical practice guideline recommends that providers routinely evaluate any patient presenting with complaints that may represent acute aortic syndrome (AAS) to establish a pretest risk of disease that can then be used to guide diagnostic decisions.

- Establishing a pretest risk of disease includes specific questions about risk factors and pain features, as well as a focused examination to identify findings that are associated with AAS.

- No further testing is suggested in those without any risk factors, pain features and findings on physical examination associated with AAS.

- In those with a moderate risk for AAS, a normal result of D-dimer testing is reasonable to reduce probability of AAS; in patients with a low or high probability of the condition, the use of D-dimer is not recommended.

- Expedited electrocardiogram-gated computed tomography of the aorta is recommended in those with a high probability for AAS.

Society of Cardiology (2014)..$^{20,21}$ However, there is still considerable variation in how clinicians investigate for AAS in Canada. ${ }^{17}$ This variation is likely multifactorial but may be a result of lack of key stakeholder involvement in the development of the guidelines or the difference in threshold for investigation within the Canadian health care system. ${ }^{22,23}$

The aims of this guideline are to update the available guideline recommendations with current evidence; include key stakeholders to allow interpretation of the evidence in context of values and preferences; and make practice recommendations that are applicable to the Canadian health care system. The full guideline, including supplemental documents, is available at Appendix 1 (www.cmaj.ca/lookup/suppl/doi:10.1503/cmaj.200021/-/DC1). 


\section{Scope}

The purpose of this guideline is to provide evidence-based recommendations about the diagnostic evaluation of patients with suspected AAS. Our definition of AAS does not include ruptured or leaking aortic aneurysms. This guideline is not applicable to pregnant patients, patients with recent $(<24 \mathrm{hr})$ cocaine use or patients younger than 18 years. The primary studies used to generate the recommendations either did not include these patients or did not report on their inclusion; therefore, the diagnostic accuracy of signs and symptoms for AAS are unknown within these populations.

We present diagnostic pathways based on the expected prevalence of AAS and provide guidance on testing based on clinically relevant and accepted thresholds for diagnostic error. The recommendations are further guided by principles of minimizing radiation exposure and the required number of diagnostic tests to meet the accepted thresholds, where possible. This guideline is intended to aid clinicians in selecting optimal diagnostic test(s) that are more likely to result in a diagnostic result, reduce the number of diagnostic tests and minimize exposure to radiation.

The target audience includes patients, emergency medicine physicians, family physicians, internists, radiologists, vascular surgeons, cardiothoracic surgeons, critical care physicians and decision-makers.

\section{Recommendations}

The 4 recommendations in this guideline are divided into the following categories: assessment of pretest probability (risk factors, pain features, clinical suspicion and alternative diagnoses, and physical examination) and diagnostic strategy (low, medium and high pretest probability) (Table 1 ). The assessment of quality of studies informing this guideline is available in Appendix 2, at www.cmaj.ca/lookup/suppl/doi:10.1503/cmaj.200021/-/DC1.

The rationale behind optimal test thresholds and the prevalence estimates used in Bayesian modelling (as described in the Methods section, below) can be found in the full guideline (Appendix 1). Modifications to the American Heart Association and European Society of Cardiology guidelines are highlighted in the description of the recommendations below and in Table 3 .

Table 2 and Figure 1 provide a framework for assessment of pretest probability and suggestions for further testing at moderate or high risk for AAS. Pretest probability is defined as the probability of a patient having the target disorder before a diagnostic test result is known. See Appendix 2 for the definitions used for each high-risk clinical finding associated with AAS.

\section{Assessment of pretest probability}

The committee recommends that providers routinely evaluate any patient presenting with complaints that may represent AAS, to establish a pretest risk of disease that can then be used to guide diagnostic decisions. This process should include specific questions about risk factors and pain features, as well as a focused examination to identify findings that are associated with AAS (strong recommendation based on low certainty in the evidence of effects on clinical outcomes and moderate certainty in the evidence of diagnostic accuracy studies).
The committee suggests using historical pain, risk factors and physical examination findings to define a patient as having a low ( $\leq 0.5 \%)$, moderate $(0.5 \%-5 \%)$ or high (> 5\%) probability for AAS (conditional recommendation based on low certainty in the evidence of effects on clinical outcomes and low certainty in the evidence of diagnostic accuracy studies) (Table 2).

\section{Risk factors}

Risk factors for AAS include connective tissue disease, aortic valve disease, recent aortic manipulation, aortic aneurysm (thoracic or abdominal, on chest radiograph, history or bedside echocardiography) and family history of AAS.

The European Society of Cardiology and American Heart Association guidelines on AAS specifically refer to thoracic aortic aneurysms as a risk factor. However, the International Registry of Acute Aortic Dissection database, a multicentre registry that includes more than 4000 cases, states that a known aortic aneurysm, either thoracic or abdominal, is associated with AAS. ${ }^{24}$ Therefore, we updated the definition of aortic aneurysm in our assessment of pretest probability to include both abdominal and thoracic aneurysms as risk factors.

Patients may not be aware of the presence of an aortic aneurysm. Bedside ultrasound has been found to be accurate in the diagnosis of abdominal aortic aneurysms. ${ }^{25} \mathrm{~A}$ multicentre prospective observational study including 839 patients (very lowquality evidence, downgraded for risk of bias and indirectness) showed moderate diagnostic accuracy (sensitivity 0.60 , 95\% confidence interval $[\mathrm{Cl}] 0.51-0.68$; specificity $0.85,95 \% \mathrm{Cl} 0.83-0.88$ ) for assessment of aneurysmal dilation of the aortic root. ${ }^{26}$ Bedside ultrasound is operator dependent; therefore, the diagnostic accuracy may vary depending on the level of training. However, it is reasonable to examine those who are presenting with symptoms suggestive of AAS for the presence of an abdominal or thoracic aortic aneurysm in the visible portions of the aorta.

We found 7 observational (prospective and retrospective) studies including 659 patients (very low- or low-quality evidence, downgraded for substantial heterogeneity and imprecision) that suggest chest radiograph may also be helpful (sensitivity 0.79, $95 \% \mathrm{Cl} 0.70-0.85$; specificity $0.56,95 \% \mathrm{Cl} 0.41-0.70$ ) in suggesting a possible thoracic aortic dilation. ${ }^{27-32} \mathrm{~A}$ systematic review of 9 studies ( $n=551$ ) found clinical examination is not sufficient in diagnosing rapid aortic expansion or a leaking, ruptured or dissecting aortic aneurysm. ${ }^{33}$ Therefore, if a patient presents with pain and a known or newly diagnosed aortic aneurysm, they should undergo advanced imaging. This is a modification of the European Society of Cardiology and American Heart Association guidelines (Table 3).

\section{High-risk pain features}

High-risk pain features for AAS include abrupt-onset or thunderclap pain, severe or worst-ever pain, tearing or ripping pain, migrating or radiating pain.

The American Heart Association guideline recommends that high-risk pain be defined as pain that is either severe or abrupt in onset, with a tearing, ripping, sharp or stabbing characteristic. Rogers and colleagues modified this recommendation to define 
high-risk pain as any abrupt, severe, tearing or ripping pain. ${ }^{34}$ These modifications formed the basis of the high-risk pain section of the acute aortic dissection detection risk score. This tool was the result of expert consensus and was incorporated into the European Society of Cardiology guideline.

In contrast, the committee agreed that a patient with severe pain versus a patient with severe, abrupt-onset, tearing pain were not at the same pretest probability for AAS. We also thought that an increasing number of high-risk pain features was associated with an increased pretest probability for AAS; however, this was based entirely on expert consensus as we found no direct evidence on systematic review of the literature.

\section{Clinical suspicion or alternative diagnosis}

Clinician suspicion for AAS or an alternative diagnosis is currently part of the American Heart Association and European Society of Cardiology guidelines; however, these guidelines suggest that consideration of an alternative diagnosis should take place after

Table 1 (part 1 of 2): Recommendations for assessment of pretest probability and diagnostic strategy in acute aortic syndrome

\section{Recommendation}

\section{Assessment of pretest probability}

The committee recommends that providers routinely evaluate any patient presenting with complaints that may represent AAS, to establish a pretest risk of disease that can then be used to guide diagnostic decisions. This process should include specific questions about risk factors and pain features, as well as a focused examination to identify findings that are associated with AAS.

The committee suggests using historical pain, risk factors and physical examination findings to define a patient as low $(\leq 0.5 \%)$, moderate $(0.5 \%-5 \%)$ or high (> 5\%) probability for AAS (Table 2 ).

\section{Strength of recommendation and certainty of evidence}

Technical remarks:

- An absence of any high-risk historical, risk factor or physical examination findings places the patient in a population with a very low prevalence for AAS.

- The panel recognized that different clinical features have different strengths of association with AAS.

- Clinical suspicion for an alternative diagnosis or for AAS is important in assessment of pretest probability.

- Patients with a low risk of AAS and no alternative diagnosis are still considered low risk.

- The panel suggests the presence of any clinical features strongly associated with AAS (hypotension, pulse deficit, neurologic deficit, new murmur of aortic regurgitation, aortic aneurysm) places the patient in a higher probability population.

- Clinical suspicion for an alternative diagnosis is useful in a moderate-risk group, but AAS should still be considered in the presence of multiple clinical features for AAS even if a suspicion for an alternative diagnosis exists.

- This assessment strategy assumes that the history and physical examination are carried out under optimal conditions in an oriented patient compliant to physical examination. If the patient is unable to provide an accurate history, pretest probability assessment will be affected.

\section{Diagnostic strategy}

\section{Low pretest probability}

The committee suggests no further testing in a population with a low pretest probability (prevalence of AAS of $\leq 0.5 \%$ ).

Technical remarks:

- There are no prospectively validated clinical decision rules to assess pretest probability. Estimates are based on modelling of low- or moderatequality observational studies.

- The pathway relies on the ability to obtain an accurate history. The addition of D-dimer to the diagnostic pathway lowered the probability of AAS further, but also increased the number of false-positive CT scans above ideal thresholds.

\section{Intermediate pretest probability}

The committee suggests using a strategy starting with D-dimer for excluding AAS in a population with an intermediate pretest probability (prevalence $0.5 \%-5 \%$ ), followed by ECG-gated CT in patients with a positive D-dimer test. If $\mathrm{D}$-dimer testing is not readily available, an alternative acceptable strategy includes performing ECG-gated CT alone.

The committee suggests no further testing after a negative D-dimer test in a population with an intermediate pretest probability (prevalence $0.5 \%-5 \%$ ). The panel recommends no additional testing after a negative ECG-gated $\mathrm{CT}$ in a population with intermediate pretest probability prevalence.
Conditional recommendation based on low certainty in the evidence of effects on clinical outcomes and moderate certainty in the evidence of diagnostic accuracy studies.

Conditional recommendation for D-dimer based on low certainty in the evidence of effects on clinical outcomes and moderate certainty in the evidence about diagnostic accuracy studies. Conditional recommendation for ECG-gated CT aorta based on moderate certainty in the evidence of effects on clinical outcomes and moderate certainty in the evidence from diagnostic accuracy studies.

Grading as per low pretest probability recommendation. 


\section{Intermediate pretest probability (cont'd)}

Technical remarks:

- There are no prospectively validated clinical decision rules to assess pretest probability. Estimates are based on modelling of low- or moderatequality observational studies.

- A decision to start with D-dimer testing assumes the results will be obtained in a timely manner and that the cost of D-dimer screening is offset by avoiding unnecessary ECG-gated CT aorta or transfer of patients at moderate pretest probability for AAS. If the D-dimer strategy is followed, a highly sensitive D-dimer assay is required.

- A negative D-dimer test (i.e., $<500 \mathrm{ng} / \mathrm{mL}$ ) indicates a low probability $(<0.5 \%)$ of AAS and no additional testing is required.

- Caution should be used in patients presenting with > $24 \mathrm{~h}$ of symptoms as D-dimer can be falsely negative. Intramural hematoma without connection to circulation can lead to a false-negative D-dimer. D-dimer has limited utility in hospitalized patients and in certain patient populations (postsurgical, pregnant) because of the high frequency of positive D-dimer results with standard thresholds.

- The likelihood of a positive D-dimer test (>500 ng/mL) increases with age; however, there are no studies reporting on the diagnostic accuracy of age-adjusted D-dimer testing for the diagnosis of AAS. Therefore, the standard threshold of $>500 \mathrm{ng} / \mathrm{mL}$ was used to indicate a positive test.

- ECG-gated CT of the aorta is the next step after a positive D-dimer result. AAS is a time-sensitive condition and CT should be expedited. The preference is for an ECG-gated scan. A negative ECG-gated CT rules out AAS.

The strategy assumes that test results are obtained under optimal conditions. Suboptimal CT results may require repeat testing or an alternate strategy.

\section{High pretest probability}

The committee recommends using a strategy starting with ECG-gated CT for assessing patients suspected of having AAS in a population with a high pretest probability (prevalence $\geq 5 \%$ ).

The committee suggests not using a D-dimer test in a population with a high pretest probability (prevalence $\geq 5 \%$ ).

Technical remarks:

- There are no prospectively validated clinical decision rules to assess for a pretest probability of $>5 \%$. Estimates are based on modelling of lowor moderate-quality observational studies.

- CT should be ECG gated to avoid motion artifacts especially of the aortic root and ascending aorta. It is important to scan with and without contrast to rule out an intramural hematoma. Reconstruction to thinner slices $(<1 \mathrm{~mm})$ and multiplanar reconstruction including sagittal oblique for thoracic aorta are recommended.

- The strategy assumes that test results are obtained under optimal conditions. Suboptimal CT results from improper technique (e.g., no gating, only unenhanced study or venous phase scanning) may require repeat testing.

- If CT is not feasible (e.g., contrast media allergy, severe renal impairment, or unavailability), MRI or TEE may be acceptable.

- In cases where clinical suspicion for AAS remains high with a negative initial CT, repeat ECG-gated CT should be considered. If repeat CT with proper technique is not feasible, additional testing with TEE or MRI may be considered.

Note: $\mathrm{AAS}=$ acute aortic syndrome, $\mathrm{CT}=$ computed tomography, $\mathrm{ECG}=$ electrocardiogram, MRI = magnetic resonance imaging, $\mathrm{TEE}=$ transesophageal echocardiography.

the assessment of pretest probability. In contrast, committee members agreed that this happens concurrently with assessment of historical risk features and should be included in initial pretest probability assessment. Results from a national survey supported this view, with respondents rating likelihood of AAS or of an alternative diagnosis as extremely important in the assessment of pretest probability, ranking higher than any pain characteristic, risk factor or physical examination finding. ${ }^{35}$

There is no formal assessment of the diagnostic accuracy of clinical suspicion for AAS. We performed a systematic review and meta-analysis of studies reporting on misdiagnosis of AAS and found 12 studies with 3433 patients (very low-quality evidence, downgraded for variable patient population, definition of missed cases and reference standard among studies, along with inconsistency, imprecision and indirectness ${ }^{6,7,9-11,13-16,36-38}$ [see Appendix 2 for more details].) The results of this meta-analysis (sensitivity $0.79,95 \% \mathrm{Cl} 0.70-0.87$; specificity $0.63,95 \% \mathrm{Cl} 0.60-0.65$ ) were incorporated into the summary estimate of diagnostic accuracy in Bayesian modelling (Appendix 1). Incorporating clinical suspicion into pretest probability assessment allowed for an improvement in specificity while keeping the miss rate below an acceptable threshold. Care should be taken if the alternative diagnosis being considered is acute coronary syndrome, stroke or an unconfirmed pulmonary embolism. These are the most common initial diagnoses in patients with a missed AAS. 4,9,10,13,16

\section{Physical examination}

High-risk physical examination findings for AAS include new aortic regurgitation (auscultated murmur or bedside echocardiography), pulse deficit, neurologic deficit, hypotension or pericardial effusion on bedside echocardiography.

The American Heart Association and European Society of Cardiology guidelines report bilateral blood pressure differential as a high-risk feature for AAS. We found no direct studies exploring 
Table 2: Clinical decision aid for assessing pretest probability and conditional recommendations for investigation at a low-risk, moderate-risk and high-risk pretest probability*

Risk assessment category

Characteristic

Score

\section{Risk factors}

- Connective tissue disease

No risk factors

- Aortic valve disease

- Recent aortic manipulation

- Family history of AAS

- Aortic aneurysm

\section{Pain features}

- Severe or worst ever

- Thunderclap or abrupt

- Tearing or ripping

- Migrating or radiating

Any nonaneurysmal risk factors

Aortic aneurysm

No high-risk pain features

1 or 2 high-risk pain features

3 or more high-risk pain features

No high-risk physical examination findings

Any high-risk physical examination findings

Suspicion for an alternative diagnosist‡

Unsure

AAS the most likely diagnosis
0

$-1$

0

\section{Score results}

0: Low-risk probability $(<0.5 \%)$ - no further investigations

1: Moderate-risk probability $(0.5 \%-5 \%)-D$-dimer testing

$\geq 2$ : High-risk probability $(>5 \%)-$ ECG-gated CT aorta

Note: $\mathrm{AAS}=$ acute aortic syndrome, $\mathrm{CT}$ = computed tomography, $\mathrm{ECG}=$ electrocardiogram

*Includes patients presenting with symptoms or signs suggestive of acute aortic syndrome (chest, abdominal or back pain) or perfusion deficit (central nervous system, cardiac, mesenteric, limb), syncope, blood pressure differential $>20 \mathrm{~mm} \mathrm{Hg}$, systolic blood pressure $>180 \mathrm{~mm} \mathrm{Hg}$; excludes patients who are $<18$ years or pregnant or those with recent trauma or cocaine use.

tIf acute coronary syndrome is suspected and there are high-risk pain features or physical examination findings or risk factors for AAS, consider chest radiograph, point-of-care ultrasound and possibly D-dimer testing.

flf pulmonary embolism is suspected and there are high-risk pain features or physical examination findings or risk factors for AAS, hold anticoagulation until confirmation.

its independent association with AAS. Multiple studies analyzed it as a composite variable with pulse deficit or blood pressure differential. ${ }^{39}$ We identified only 1 case-control study that showed bilateral blood pressure differential was significantly associated with AAS; however, it did not improve diagnostic accuracy in addition to pulse deficit. ${ }^{40}$

As much as $20 \%$ of the general population will have a blood pressure differential. ${ }^{41}$ The committee agreed that this finding may be useful to raise suspicion for AAS; thus, any patient presenting with a blood pressure differential should be assessed for their pretest probability of AAS. In the context of other pain, risk factors and physical examination findings, it likely does not add any benefit in assessment of pretest probability. Therefore, we removed bilateral blood pressure differential from the high-risk physical examination findings (Table 3 ).

A single-centre study of 839 patients (very low-quality evidence, downgraded for risk of bias and indirectness) suggests that bedside ultrasound is capable of diagnosing aortic regurgitation in AAS (sensitivity $0.1,95 \% \mathrm{Cl} 0.06-0.16$; specificity 0.93 , $95 \% \mathrm{Cl} 0.91-0.95) .{ }^{26}$ Diagnostic accuracy of bedside ultrasound for aortic regurgitation may vary between providers. This variation is also seen with clinical examination. Auscultation has moderate-to-low inter-rater reliability and diagnostic accuracy. ${ }^{42,43}$ Therefore, the committee agreed that point-of-care ultrasonographic evidence of new aortic regurgitation in a patient presenting with symptoms suggestive of AAS is a highrisk examination finding, in addition to auscultation of a murmur consistent with aortic regurgitation. The use of point-ofcare ultrasound is meant to augment physical examination; therefore, if a provider is not trained in its use, it is simply omitted from pretest probability assessment. The addition of pointof-care ultrasonography is an update to the American Heart Association guideline, but is present in the European Society of Cardiology guideline (Table 3). 
Table 3 : Comparison of Canadian guideline recommendations to American Heart Association and European Society of Cardiology guidelines*

\begin{tabular}{|c|c|}
\hline Characteristics & Canadian guideline \\
\hline \multicolumn{2}{|l|}{ Clinical features } \\
\hline Aortic aneurysm & $\begin{array}{l}\text { Definition } \\
\text { - History of thoracic or abdominal } \\
\text { aortic aneurysm } \\
\text { - Bedside ultrasound with aortic } \\
\text { dilation } \\
\text { - Widened mediastinum on chest } \\
\text { radiograph } \\
\text { Recommendation } \\
\text { - If present in isolation, high } \\
\text { probability }\end{array}$ \\
\hline
\end{tabular}

\section{High-risk pain features Definition}

Blood pressure

differential

$>20 \mathrm{~mm} \mathrm{Hg}$

Aortic regurgitation

\section{Pulse deficit}

Neurological deficit

Hypotension

Pericardial effusion

\section{Pretest probability}

Low

No further testing

Intermediate

D-dimer

High assessment

\section{Definition} with pain

\section{Definition} a shock index $>1$

\section{Recommendation}

Chest, back or abdominal pain described as:

- Abrupt or thunderclap

- Severe or worst ever

- Tearing or ripping

- Migrating or radiating

Recommendation

- None present, low probability

- 1 or 2 , intermediate probability

- 3 or more, high probability

Recommendation

Not part of pretest probability

New murmur or aortic regurgitation

\section{Recommendation}

If present in isolation, high probability (ECG-gated CT aorta)

\section{Recommendation}

If present in isolation, high probability

\section{Recommendation}

If present in isolation, high probability

Systolic blood pressure $<90 \mathrm{~mm} \mathrm{Hg}$ or

\section{Recommendation}

If present in isolation, high probability

If present in isolation, high probability

\section{Definition}

Chest, back or abdominal pain described as:

- Abrupt onset or severe; and

- Tearing or ripping, or sharp or stabbing

Recommendation

- None present, low probability

- If pain described as above, intermediate probability

\section{Recommendation}

If present in isolation, intermediate probability

\section{Definition}

New murmur or aortic regurgitation with pain

\section{Recommendation}

If present in isolation, intermediate probability

\section{Recommendation}

If present in isolation, intermediate probability

\section{Recommendation}

If present in isolation, intermediate probability

\section{Definition}

Systolic blood pressure $<90 \mathrm{~mm} \mathrm{Hg}$

\section{Recommendation}

If present in isolation, intermediate probability

Not included in pretest probability assessment

Consider CT aorta if no alternative diagnosis

Expedited $\mathrm{CT}$ aorta if no alternative diagnosis

ECG-gated CT aorta

European Society of Cardiology ${ }^{21}$

Definition

- History of thoracic aneurysm Recommendation

- If present in isolation, low probability

\section{Definition}

Chest, back or abdominal pain described as any of the following:

- Abrupt

- Severe

- Tearing or ripping

Recommendation

- None present, low probability

- 1 or more present in isolation, low probability

\section{Recommendation}

If present in isolation, low probability

\section{Definition}

New murmur or aortic regurgitation with pain

\section{Recommendation}

If present in isolation, low probability

\section{Recommendation}

If present in isolation, low probability

\section{Recommendation}

If present in isolation, low probability

\section{Definition}

Systolic blood pressure $<90 \mathrm{~mm} \mathrm{Hg}$

\section{Recommendation}

If present in isolation, low probability

\section{Recommendation}

If present in isolation, high probability

Perform D-dimer, chest radiograph, transthoracic echocardiography

No intermediate probability category

ECG-gated CT aorta

Note: $\mathrm{ECG}$ = electrocardiogram, $\mathrm{CT}$ = computed tomography.

* "If present in isolation" refers to the presence of the sign or symptom in isolation. If the sign or symptom is present in context of other high-risk features for AAS, this changes the probability. See the full guideline (Appendix 1) for further information. Definitions for signs and symptoms associated with AAS are described in Appendix 2. 


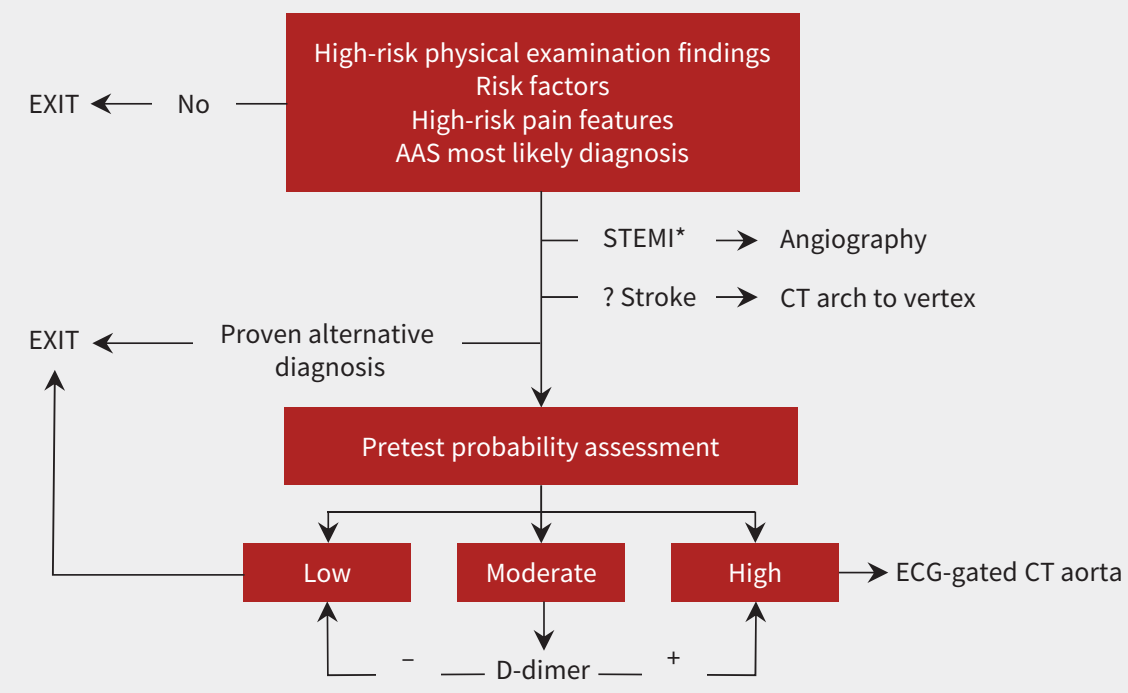

Figure 1: Diagnostic pathway for patients presenting with clinical features suggestive of AAS. *STEMI can co-exist with AAS; care should be taken to elicit all relevant risk factors for AAS and high-risk pain features and physical examination findings that might raise the suspicion for AAS. Note: AAS = acute aortic syndrome, ECG = electrocardiogram, CT = computed tomography, STEMI = ST-elevation myocardial infarction.

\section{Diagnostic strategy}

\section{Low pretest probability}

The committee suggests no further testing in a population with a low pretest probability (prevalence of AAS of $\leq 0.5 \%$ ) (conditional recommendation based on low certainty in the evidence of effects on clinical outcomes and moderate certainty in the evidence of diagnostic accuracy studies).

\section{Intermediate pretest probability}

The committee suggests using a strategy starting with D-dimer testing for excluding AAS in a population with an intermediate pretest probability (prevalence $0.5 \%-5 \%$ ), followed by ECG-gated CT in patients with a positive $D$-dimer test. If D-dimer testing is not readily available, an alternative acceptable strategy includes performing ECG-gated CT alone (conditional recommendation for $D$-dimer based on low certainty in the evidence of effects on clinical outcomes and moderate certainty in the evidence about diagnostic accuracy studies; conditional recommendation for ECG-gated CT based on moderate certainty in the evidence of effects on clinical outcomes and moderate certainty in the evidence from diagnostic accuracy studies).

The committee suggests no further testing after a negative $D$-dimer test in a population with an intermediate pretest probability (prevalence $0.5 \%-5 \%$ ). The panel recommends no additional testing after a negative ECG-gated CT in a population with an intermediate pretest probability (prevalence $0.5 \%-5 \%$ ) (grading as per low pretest probability recommendation).

\section{High pretest probability}

The committee recommends using a strategy starting with ECGgated CT for assessing patients suspected of having AAS in a population with a high pretest probability (prevalence $\geq 5 \%$ ) (strong recommendation for ECG-gated CT based on moderate certainty in the evidence of effects on clinical outcomes and moderate certainty in the evidence of diagnostic accuracy studies).

The committee suggests not using a D-dimer test in a population with a high pretest probability (prevalence $\geq 5 \%$ ) (conditional recommendation against D-dimer based on low certainty in the evidence of effects on clinical outcomes and moderate certainty in the evidence of diagnostic accuracy studies).

\section{D-dimer testing}

The recommendations for D-dimer testing are based on a metaanalysis of 22 studies with 3860 patients (Appendix 2). According to the QUADAS-2 (quality assessment of diagnostic accuracy studies) tool, 19/22 of the included studies had at least 1 domain rated as high risk of bias. The evidence was rated as very low for specificity $(0.60,95 \% \mathrm{Cl} 0.48-0.71)$ and moderate for sensitivity (0.95, 95\% Cl 0.90-0.99). There are 2 other meta-analyses that included only 5 of the 22 articles we included in the meta-analysis; their QUADAS assessments found a low or unclear risk of bias in most domains, leading to downgrading of the level of certainty. ${ }^{44,45}$

\section{ECG-gated CT of the aorta}

Acute aortic syndrome is a time-sensitive emergency and if a patient is deemed at high risk, an ECG-gated CT of the aorta 
should be expedited. We found 1 meta-analysis with a total of 3 studies. Although a small number of patients $(n=126)$ were included, there were no serious limitations in indirectness, inconsistency, imprecision or risk of bias.

Whenever available, ECG gating (prospective) should be used for imaging of suspected acute aortic syndrome, to avoid motion artifacts especially at the aortic root and ascending aorta. Scanning of the thoracic aorta without contrast is important to rule out intramural hematoma. Reconstruction to thinner slices $(<1 \mathrm{~mm})$ and multiplanar reconstruction including sagittal oblique for thoracic aorta are suggested.

\section{Methods}

We chose to adapt existing high-quality clinical practice guidelines previously developed by the American Heart Association and European Society of Cardiology using the Grading of Recommendations Assessment, Development and Evaluation (GRADE)-ADOLOPMENT (combined use of adoption, adaptation, and de novo development of recommendations) approach. ${ }^{20,21,46}$ The overall guideline development process - including funding of the work, formation of participating groups, management of competing interests, internal and external review, and organizational approval - was guided by the Guidelines International Network-McMaster Guideline Development Checklist. ${ }^{25}$ We structured our process to meet the recommendations for trustworthy guidelines by the National Academy of Medicine and the Guidelines International Network. ${ }^{47-50}$ The full details of the guideline methods are available in our complete guideline document (Appendix 1).

\section{Composition of participating groups}

We recruited 21 people from across Canada to take part in developing the guideline as members of the guideline advisory committee. The advisory committee included emergency physicians and nurses, cardiothoracic and vascular surgeons, cardiac anesthesiologists, critical care physicians, radiologists and 2 patients. We selected members based on their expertise and professional and geographic diversity, as well as practice environment (academic, community, rural or remote). Methodologists with expertise in evidence appraisal and guideline development were also included (R.O., J.Y., K.Y., E.L.). From members of the advisory committee, we appointed a guideline chair (R.O.) and a guideline coordination panel (S.M., D.A., P.J.).

We prepared and revised the guideline through an iterative process consisting of feedback and discussions until we achieved consensus. The advisory committee communicated through multiple teleconference meetings, emails and a 1-day, in-person meeting in late 2018. The coordination panel developed and graded the recommendations and assessed the certainty in the supporting evidence following the GRADE approach. ${ }^{51,52}$ We used the in-person meeting to discuss the evidence-to-decision frameworks and come to consensus on the recommendations.
Formulating specific clinical questions and determining outcomes of interest

The coordination panel used the GRADEpro Guideline Development Tool (www.gradepro.org) ${ }^{51}$ to brainstorm, then prioritize the following questions addressed by this guideline:

1. What is the optimal test threshold (i.e., the likelihood of the disease where the benefits of testing outweigh the harms) for low-, moderate- or high-risk probability for AAS?

2. What is the optimal pretest probability assessment for AAS?

3. In a patient population with a low clinical probability of AAS, what is the optimal diagnostic strategy to evaluate for suspected AAS?

4. In a patient population with an intermediate clinical probability of AAS, what is the optimal diagnostic strategy to evaluate for suspected AAS?

5. In a patient population with a high clinical probability of AAS, what is the optimal diagnostic strategy to evaluate for suspected AAS?

After the coordination panel prioritized the questions, the chair developed diagnostic pathways that were refined through an iterative process with input from the panel. The diagnostic pathways were based on signs and symptoms and additional testing for AAS.

We used these pathways to guide the evidence synthesis and recommendations. The coordination panel selected outcomes of interest for each question a priori, following the approach described in detail elsewhere. ${ }^{52}$ In brief, the coordination panel brainstormed all possible outcomes, then rated their relative importance for decision-making according to the GRADE approach. ${ }^{53}$ The coordination panel rated the following outcomes as critical for decision-making across the AAS diagnosis questions: missed diagnosis, increase in $\mathrm{CT}$ use, increase in D-dimer use and unnecessary transfer, in addition to the diagnostic accuracy outcomes (i.e., false-positive, false-negative, true-positive and true-negative test results).

The coordination panel in conjunction with the patient representatives placed higher value on reducing missed diagnosis for AAS without a substantial increase in radiation exposure to the patient if the diagnosis could be obtained using alternate methods with less radiation exposure. This decision is supported by a recent article assessing patient research priorities for AAS. $^{7}$ In most instances, there was no direct evidence assessing the effect of using 1 diagnostic pathway versus another on patient outcomes or directly comparing the accuracy of different diagnostic pathways. To make judgments about health care and patientrelated desirable and undesirable effects, the coordination panel reviewed all the potential pathways and determined the downstream consequences related to a particular test result, as well as the complications of testing itself. This review affected the degree of certainty in the recommendations.

\section{Evidence review}

To support the guideline, systematic reviews were performed (R.O., S.M., N.F.) for studies of diagnostic accuracy and prevalence estimates, as per the Cochrane Handbook for Systematic Reviews of Interventions (www.handbook.cochrane.org). We 
searched the electronic databases MEDLINE and Embase (19682018) with the assistance of a librarian, using a combination of Medical Subject Headings (MeSH) terms and keywords; there was no language restriction. The searches were updated March 2019 (no new studies were identified). For the full search strategy, see the evidence-to-decision framework document titled "What is the optimal assessment of pretest probability for AAS?" in Appendix A-1 of the full guideline (Appendix 1).

We used the QUADAS-2 tool to assess risk of bias for included studies. ${ }^{54}$ We evaluated the quality of the evidence that met inclusion criteria following the GRADE approach, based on the following domains: risk of bias, precision, consistency and magnitude of the estimate of effects, directness of the evidence and risk of publication bias. ${ }^{55}$

We categorized the certainty of evidence into 4 levels, ranging from very low to high. ${ }^{53,55,56}$ In the absence of any data, the level of evidence was rated as very low, because it was based on expert consensus.

There is very little published evidence on diagnostic pathways related to AAS; therefore, the coordination panel developed simple Bayesian models to calculate test accuracy of different pathways with a different combination of signs, symptoms and tests. The model included data obtained from the systematic reviews for AAS prevalence and the pooled diagnostic test accuracy for different tests. We used this method to present the test results explicitly, while considering different prevalence estimates, multiple potential diagnostic pathways and different assumptions about the performance of the tests in different populations. We summarized the predicted overall test strategy results from the model and the quality of evidence assessed using the GRADE approach in evidence tables, which allowed for a comparison of the benefits and harms of different diagnostic strategies.

\section{Development of recommendations}

For each guideline question, we created a GRADE "evidence-todecision" framework, using the GRADEpro Guideline Development Tool (www.gradepro.org). ${ }^{56-58}$ The evidence-to-decision table summarized the results of systematic reviews of the literature that were performed specifically for this guideline ${ }^{58}$ (see full guideline at Appendix 1). The evidence-to-decision table addressed effects of interventions including potential benefits and harms of the diagnostic test, test accuracy, resource utilization, values and preferences (e.g., relative importance of outcomes), equity, feasibility and acceptability.

A 1-day in-person meeting for advisory committee members was held in Sudbury, Ontario, on Nov. 23, 2018. The meeting was used to discuss the proposed diagnostic pathways and evidenceto-decision frameworks and come to consensus with regard to the strength of recommendations.

Recommendations were based on the balance of benefits and harms, the quality of evidence, and patient values and preferences, as per GRADE methodology. The initial draft recommendations were prepared by R.O. Interpretation of the strength of recommendations can be found in Box 1 . Consensus was achieved through discussion. Initial plans were to use online
Box 1: Interpretation of strong and conditional recommendations

The strength of a recommendation is expressed as either strong ("the guideline panel recommends ..."), or conditional ("the guideline panel suggests ...") and is interpreted as shown below. ${ }^{59}$

\section{Strong recommendation}

- For patients: most individuals in this situation would want the recommended course of action, and only a small proportion would not.

- For clinicians: most individuals should receive the intervention or test. Formal decision aids are not likely to be needed to help individual patients make decisions consistent with their values and preferences.

- For policy-makers: the recommendation can be adopted as policy in most situations. Adherence to this recommendation according to the guideline could be used as a quality criterion or performance indicator.

- For researchers: the recommendation is supported by credible research or other convincing judgments that make additional research unlikely to alter the recommendation. On occasion, a strong recommendation is based on low- or very low-certainty evidence. ${ }^{60}$

\section{Conditional recommendation}

- For patients: the majority of individuals in this situation may want the suggested course of action, but many may not. Decision aids may be useful in helping patients to make decisions consistent with their individual risks, values and preferences.

- For clinicians: different choices will be appropriate for individual patients and clinicians must help each patient arrive at a management decision consistent with their values and preferences through shared decision-making. Decision aids may be useful in helping individuals to make decisions consistent with their individual risks, values and preferences.

- For policy-makers: policy-making will require substantial debate and involvement of various stakeholders. Performance measures about the suggested course of action should focus on whether an appropriate decision-making process is duly documented.

- For researchers: this recommendation is likely to be strengthened (for future updates or adaptation) by additional research. An evaluation of the conditions and criteria (and the related judgments, research evidence and additional considerations) that determined the conditional (rather than strong) recommendation will help identify possible research gaps.

software for anonymous voting using a modified Delphi process; however, the coordination panel and the advisory committee decided that they would prefer open discussion. Unanimous consensus was reached on all draft recommendations.

\section{Clinical decision aid}

We developed a clinical decision aid to compile the recommendations into a single tool (Table 2). We combined the Bayesian models for each sign and symptom together with our identified values and preferences and defined thresholds for advanced imaging or no further testing. We used thresholds for investigation 
informed by a national survey of emergency physicians to define low $(<0.5 \%)$, intermediate $(0.5 \%-5 \%)$ or high $(<5 \%)$ pretest probability groups. ${ }^{35}$

Using Bayesian models, signs and symptoms were applied to a prevalence of $2 \%$ to generate a post-test probability. If the post-test probability was $<0.5 \%$, the clinical variable was assigned a value of 0 ; if $0.5 \%-5 \%$, a value of 1 ; and if $>5 \%$, a value of 2. Further investigations (i.e., D-dimer testing, ECG-gated CT aorta) were then applied to the post-test probabilities to determine their utility. See the full guideline for the method used to generate the prevalence estimates and to define the test thresholds (Appendix 1).

\section{External review}

The draft recommendations were distributed to more than 300 emergency medicine physicians, radiologists and surgeons for review in October 2019. The goal of the review was to obtain stakeholder feedback on the interpretation of the evidence, the strength of the recommendations and document layout.

Key stakeholder organizations also reviewed the guideline and supporting documents: the Canadian Association of Emergency Physicians, the Canadian Society of Cardiac Surgery, the Canadian Society of Vascular Surgery and the Canadian Society of Thoracic Radiology.

The coordinating panel revised the document to address pertinent comments by the stakeholder organizations and external reviewers. The advisory committee then reviewed and approved these revisions. Each stakeholder organization provided endorsement of the final document.

\section{Management of competing interests}

Development of this guideline was wholly funded by the Alternate Funding Plan grant of the Northern Ontario Academic Medical Association, a nonprofit academic medical association. Members of the advisory committee received travel reimbursement for attendance at the in-person meeting. Members of the coordination panel received no other payments.

We managed competing interests of all participants according to recommendations of the Institute of Medicine and the Guidelines International Network. ${ }^{48,50}$ Before appointment to the advisory committee, individuals disclosed both direct (financial) and indirect (nonfinancial) interests. Members of the coordination panel and chair reviewed the disclosures and judged which interests were conflicts and should be managed. At the time of appointment, the entire advisory committee had no direct competing interests, defined as no current material interest in any commercial entity with a product that could be affected by the guideline. After the guideline was completed, this process was repeated; no additional competing interests were reported.

\section{Implementation}

Adaptation of these recommendations will likely be necessary in some circumstances; this document may serve as a basis for adaptation by local, regional or national guideline groups. For example, guideline implementation in an urban centre with 24-hour access to CT may differ from a rural or remote location that requires transfer of a patient with accompanying staff. These adaptations should be based on the associated evidenceto-decision frameworks. ${ }^{46}$

Using a theory-driven, qualitative approach, we interviewed emergency medicine physicians from 4 urban and rural hospitals to describe current practice and to identify the barriers and facilitators to a standardized approach for investigating patients for AAS (Cait Dimitrew, Northern Ontario School of Medicine, Sudbury, Ontario; unpublished data 2020). Saturation of themes was achieved after 9 physician interviews.

We identified 3 barriers to implementation of this guideline: use of D-dimer and knowledge of rationale for its use in AAS is not widespread; the decision aid in the guideline was not in alignment with current practice or physician understanding of risk factors; and the complexity of the suggested decision aid in the guideline was a potential barrier to accurate application and desired outcomes.

We also identified 3 facilitators: publishing the guideline recommendations and decision aid would increase use and acceptability; availability of the decision aid and recommendations as an online tool would increase use; and clinicians were confident that the recommendations and decision tool would support clinical decision-making and risk stratification and had the potential to reduce resource use (e.g., CT), particularly in rural settings.

\section{Suggested performance measures}

Based on consensus of the advisory committee, we developed a suggested set of performance measures to accompany this guideline for consideration by providers and policy-makers: proportion of missed cases of AAS, time to diagnosis of AAS, emergency department disposition time, proportion of patients receiving a CT aorta, proportion of patients with D-dimer testing, and proportion of patients transferred for imaging or consultation.

\section{Updates}

The guideline coordination panel will be responsible for updating the guideline every 5 years.

\section{Other guidelines}

The recommendations in this guideline were adapted from the American Heart Association and European Society of Cardiology guidelines; comparison of the 3 guidelines is available in Table 3 . All 3 guidelines recommend further testing depending on assessment of pretest probability. However, the importance of specific clinical features and results of investigations may be interpreted differently. Additionally, the recommended testing by pretest probability varies among the guidelines. These differences are based on the interpretation of evidence and the thresholds for investigations in different practice environments.

\section{Gaps in knowledge}

The recommendations in this guideline are largely based on very low- or low-quality evidence and on modelled estimates for the 
population prevalence of AAS. The evidence base would benefit from prospective data collection of important high-risk clinical variables in order to increase the quality of evidence. Prevalence and pretest probability are continuous variables but for modelling purposes, required input of a specific population prevalence. Prevalence estimates greatly different from those used in modelling will affect the recommendations. Future studies are needed to validate the diagnostic accuracy of this guideline's recommendations across a spectrum of disease prevalence in emergency departments.

\section{Conclusion}

Acute aortic syndrome is a difficult-to-diagnose aortic emergency. We propose recommendations to aid clinicians in riskstratifying patients and, depending on risk level, suggest further investigations needed. This guideline is intended as a resource for practising clinicians, both as an evidence base and a guide to investigation for this high-risk aortic catastrophe.

\section{References}

1. Vilacosta I, San Román JA, Aragoncillo P, et al. Penetrating atherosclerotic aortic ulcer: documentation by transesophageal echocardiography. J Am Coll Cardiol 1998;32:83-9.

2. Meszaros I, Morocz J, Szlavi J, et al. Epidemiology and clinicopathology of aortic dissection: a population-based longitudinal study over 27 years. Chest 2000;117:1271-8.

3. Eslick GD, Jones M, Talley N. Non-cardiac chest pain: prevalence, risk factors, impact and consulting - a population-based study. Aliment Pharmacol Ther 2003;17:1115-24.

4. Harris KM, Strauss CE, Eagle KA, et al. Correlates of delayed recognition and treatment of acute type $A$ aortic dissection the International Registry of Acute Aortic Dissection (IRAD). Circulation 2011;124:1911-8.

5. Pape LA, Awais M, Woznicki EM, et al. Presentation, diagnosis, and outcomes of acute aortic dissection: 17-year trends from the International Registry of Acute Aortic Dissection. J Am Coll Cardiol 2015;66:350-8.

6. Asouhidou I, Asteri T. Acute aortic dissection: be aware of misdiagnosis. BMC Res Notes 2009;2:25.

7. Chua M, Ibrahim I, Neo X, et al. Acute aortic dissection in the ED: risk factors and predictors for missed diagnosis. Am J Emerg Med 2012;30:1622-6.

8. Eagle KA, Quertermous T, Kritzer GA, et al. Spectrum of conditions initially suggesting acute aortic dissection but with negative aortograms. Am J Cardiol 1986;57:322-6.

9. Hansen MS, Nogareda GJ, Hutchison SJ. Frequency of and inappropriate treatment of misdiagnosis of acute aortic dissection. Am J Cardiol 2007;99:852-6.

10. Hirata K, Wake M, Takahashi T, et al. Clinical predictors for delayed or inappropriate initial diagnosis of type A acute aortic dissection in the emergency room. PLoS One 2015;10:e0141929.

11. Kurabayashi M, Miwa N, Ueshima D, et al. Factors leading to failure to diagnose acute aortic dissection in the emergency room. J Cardiol 2011;58:287-93.

12. Li Y, Li L, Mu HS, et al. Aortic dissection and sudden unexpected deaths: a retrospective study of 31 forensic autopsy cases. J Forensic Sci 2015;60:1206-11.

13. Rapezzi C, Rocchi G, Fattori R, et al. Usefulness of transesophageal echocardiographic monitoring to improve the outcome of stent-graft treatment of thoracic aortic aneurysms. Am J Cardiol 2001;87:315-9.

14. Rosman HS, Patel S, Borzak S, et al. Quality of history taking in patients with aortic dissection. Chest 1998;114:793-5.

15. Sullivan PR, Wolfson AB, Leckey RD, et al. Diagnosis of acute thoracic aortic dissection in the emergency department. Am J Emerg Med 2000;18:46-50.

16. Zhan S, Hong S, Shan-shan L, et al. Misdiagnosis of aortic dissection: experience of 361 patients. J Clin Hypertens (Greenwich) 2012;14:256-60.

17. Ohle R, Anjum O, Bleeker $\mathrm{H}$, et al. Variation in emergency department use of computed tomography for investigation of acute aortic dissection. Emerg Radiol 2018;25:293-8.
18. Lovy AJ, Bellin E, Levsky JM, et al. Preliminary development of a clinical decision rule for acute aortic syndromes. Am J Emerg Med 2013;31:1546-50.

19. Bandali MF, Hatem MA, Appoo JJ, et al. False positive computed tomographic angiography for Stanford type A aortic dissection. Radiol Case Rep 2015;10:31-5.

20. Hiratzka LF, Bakris GL, Beckman JA, et al. 2010 ACCF/AHA/AATS/ACR/ASA/SCA/ SCAI/SIR/STS/SVM guidelines for the diagnosis and management of patients with thoracic aortic disease. J Am Coll Cardiol 2010;55:e27-129.

21. Erbel R, Aboyans V, Boileau C, et al. 2014 ESC guidelines on the diagnosis and treatment of aortic diseases. Eur Heart J 2014;35:2873-926.

22. Berdahl CT, Vermeulen MJ, Larson DB, et al. Emergency department computed tomography utilization in the United States and Canada. Ann Emerg Med 2013;62:486-94. e3.

23. Graham ID, Harrison MB. Evaluation and adaptation of clinical practice guidelines. Evid Based Nurs 2005;8:68-72.

24. Evangelista A, Isselbacher EM, Bossone $E$, et al. Insights from the International Registry of Acute Aortic Dissection: a 20-year experience of collaborative clinical research. Circulation 2018;137:1846-60.

25. Bentz S, Jones J. Accuracy of emergency department ultrasound scanning in detecting abdominal aortic aneurysm. Emerg Med J 2006;23:803-4.

26. Nazerian P, Mueller C, Vanni S, et al. Integration of transthoracic focused cardiac ultrasound in the diagnostic algorithm for suspected acute aortic syndromes. Eur Heart J 2019;24:1952-60.

27. Chan K-L. Usefulness of transesophageal echocardiography in the diagnosis of conditions mimicking aortic dissection. Am Heart J 1991;122:495-504.

28. Armstrong WF, Bach DS, Carey LM, et al. Clinical and echocardiographic findings in patients with suspected acute aortic dissection. Am Heart J 1998;136: 1051-60.

29. Ersel M, Aksay E, Kiyan S, et al. Can D-dimer testing help emergency department physicians to detect acute aortic dissections? AKD 2010;10:434-9.

30. Granato JE, Dee P, Gibson RS. Utility of two-dimensional echocardiography in suspected ascending aortic dissection. Am J Cardiol 1985;56:123-9.

31. Hartnell GG, Wakeley CJ, Tottle A, et al. Limitations of chest radiography in discriminating between aortic dissection and myocardial infarction: implications for thrombolysis. J Thorac Imaging 1993;8:152-5.

32. von Kodolitsch Y, Schwartz AG, Nienaber CA. Clinical prediction of acute aortic dissection. Arch Intern Med 2000;160:2977-82.

33. Lederle FA, Simel DL. Does this patient have abdominal aortic aneurysm? JAMA 1999;281:77-82.

34. Rogers AM, Hermann LK, Booher AM, et al. Sensitivity of the aortic dissection detection risk score, a novel guideline-based tool for identification of acute aortic dissection at initial presentation: results from the international registry of acute aortic dissection. Circulation 2011;123:2213-8.

35. Ohle R, Mclsaac S, Yan J, et al. National survey of emergency physicians on the risk stratification and acceptable miss rate of acute aortic syndrome. CJEM 2020;22:309-12.

36. Nazerian P, Mueller C, de Matos Soeiro A, et al. Diagnostic accuracy of the aortic dissection detection risk score plus D-dimer for acute aortic syndromes: the ADvISED Prospective Multicenter Study. Circulation 2018;137:250-8.

37. Nazerian P, Giachino F, Vanni S, et al. Diagnostic performance of the aortic dissection detection risk score in patients with suspected acute aortic dissection. Eur Heart J Acute Cardiovasc Care 2014;3:373-81.

38. Ohle R, Um J, Anjum $\mathrm{O}$, et al. High-risk clinical features for acute aortic dissection: a case-control study. Acad Emerg Med 2018;25:378-87.

39. Klompas M. Does this patient have an acute thoracic aortic dissection? JAMA 2002;287:2262-72.

40. Um SW, Ohle R, Perry JJ. Bilateral blood pressure differential as a clinical marker for acute aortic dissection in the emergency department. Emerg Med J 2018;35:556-8.

41. Clark CE, Campbell J, Evans P, et al. Prevalence and clinical implications of the inter-arm blood pressure difference: a systematic review. J Hum Hypertens 2006;20:923-31.

42. Mangione S, Nieman LZ. Cardiac auscultatory skills of internal medicine and family practice trainees: a comparison of diagnostic proficiency. JAMA 1997;278:717-22.

43. Patel A, Tomar NS, Bharani A. Utility of physical examination and comparison to echocardiography for cardiac diagnosis. Indian Heart J 2017;69:141-5. 
44. Asha SE, Miers JW. A systematic review and meta-analysis of D-dimer as a ruleout test for suspected acute aortic dissection. Ann Emerg Med 2015;66:368-78.

45. Cui JS, Jing ZP, Zhuang SJ, et al. D-dimer as a biomarker for acute aortic dissection: a systematic review and meta-analysis. Medicine 2015;94:e471.

46. Schünemann HJ, Wiercioch W, Brozek J, et al. GRADE Evidence to Decision (EtD) frameworks for adoption, adaptation, and de novo development of trustworthy recommendations: GRADE-ADOLOPMENT. J Clin Epidemiol 2017;81:101-10.

47. Schünemann HJ, Wiercioch W, Etxeandia I, et al. Guidelines 2.0: systematic development of a comprehensive checklist for a successful guideline enterprise. CMAJ 2014;186:E123-42.

48. Medicine Io. Clinical practice guidelines we can trust. In: Graham R, Mancher M, Wolman DM, editors. Washington (D.C.): The National Academies Press; 2011:290.

49. Qaseem A, Forland F, Macbeth F, et al. Guidelines International Network: toward international standards for clinical practice guidelines. Ann Intern Med 2012;156:525-31.

50. Schünemann HJ, Al-Ansary LA, Forland F, et al. Guidelines International Network: principles for disclosure of interests and management of conflicts in guidelines. Ann Intern Med 2015;163:548-53.

51. Brożek J, Nowak A, Kunstman P, et al. GRADEpro guideline development tool (G2DT). Version. 2014;2.

52. Guyatt GH, Oxman AD, Kunz R, et al. GRADE guidelines: 2. Framing the question and deciding on important outcomes. J Clin Epidemiol 2011;64:395-400.
53. Guyatt G, Oxman AD, Akl EA, et al. GRADE guidelines: 1. Introduction-GRADE evidence profiles and summary of findings tables. J Clin Epidemiol 2011; 64:383-94.

54. Whiting PF, Rutjes AW, Westwood ME, et al. QUADAS-2: a revised tool for the quality assessment of diagnostic accuracy studies. Ann Intern Med 2011;155:529-36.

55. Guyatt $\mathrm{GH}$, Oxman AD, Vist GE, et al. GRADE: an emerging consensus on rating quality of evidence and strength of recommendations. BMJ 2008; 336:924-6.

56. Schünemann HJ, Mustafa R, Brozek J, et al. GRADE Guidelines: 16. GRADE evidence to decision frameworks for tests in clinical practice and public health. $J$ Clin Epidemiol 2016;76:89-98.

57. Alonso-Coello P, Oxman AD, Moberg J, et al. GRADE Evidence to Decision (EtD) frameworks: a systematic and transparent approach to making well informed healthcare choices. 2: Clinical practice guidelines. BMJ 2016;353:i2089.

58. Alonso-Coello P, Schünemann HJ, Moberg J, et al. GRADE Evidence to Decision (EtD) frameworks: a systematic and transparent approach to making well informed healthcare choices. 1: Introduction. BMJ 2016;353:i2016.

59. Grades of Recommendation, Assessment, Development, and Evaluation. Canadian Task Force on Preventive Health Care; 2019. Available: https://canadiantaskforce. $\mathrm{ca} /$ methods/grade/ (accessed 2018 May 20).

60. Hultcrantz M, Rind D, Akl EA, et al. The GRADE Working Group clarifies the construct of certainty of evidence. J Clin Epidemiol 2017;87:4-13.
Competing interests: Ashish Gupta reports receiving reimbursement of travel- and stay-related expenses from the Northern Ontario Academic Medical Association grant, to attend a meeting for this work. No other competing interests were declared.

This article has been peer reviewed.

Affiliations: The Department of Emergency Medicine, Health Science North Research Institute (Ohle, Middaugh, Ansell, Scott), Northern Ontario School of Medicine, Sudbury, Ont.; Patient representative (Callaway), Alexandria, Ont.; Division of Cardiac Surgery (Rubens), University of Ottawa, Ottawa, Ont.; Division of Emergency Medicine, Department of Medicine (Yan), Western University, London, Ont.; Department of Emergency Medicine (Yadav), The Ottawa Hospital, University of Ottawa, Ottawa, Ont.; Department of Emergency Medicine (Cournoyer), Hôpital du Sacré-Coeur de Montréal, Université de Montréal, Montréal, Que.; Department of Emergency Medicine (Savage), Northern Ontario School of Medicine, Lakehead University, Thunder Bay, Ont.; Department of Vascular Surgery (Jetty), The Ottawa Hospital, University of Ottawa, Ottawa, Ont.; Division of Cardiac Surgery (Atoui), Health Sciences North, Northern Ontario School of Medicine, Sudbury, Ont.; Division of Cardiac Surgery (Atoui, Bittira), Health Sciences North, Northern Ontario School of Medicine, Sudbury, Ont.; Department of Radiology (Gupta), The Ottawa Hospital, University of Ottawa, Ottawa, Ont.; Department of Radiology (Coffey), Trillium Hospital, University of Toronto, Mississauga, Ont.; Division of Cardiac Anesthesiology (Wilson), Department of Anesthesiology and Pain Medicine, University of Ottawa Heart Institute, Ottawa, Ont.; Department of Emergency Medicine
(Bignucolo), Espanola Regional Hospital, Northern Ontario School of Medicine, Espanola, Ont.; Department of Anaesthesia (Mclsaac), Department of Critical Care, Health Science North, Northern Ontario School of Medicine, Sudbury, Ont.; Department of Emergency Medicine (Lang), Cumming School of Medicine, University of Calgary and Rockyview General Hospital (Lang), Calgary, Alta.

Contributors: All of the authors contributed to the conception and design of the work, and the acquisition, analysis, and interpretation of data. Robert Ohle drafted the manuscript. Guideline coordination panel members (Justin Yan, Krishan Yadav, Rony Atoui, David Savage) revised it critically for important intellectual content. All of the authors gave final approval of the version to be published and agreed to be accountable for all aspects of the work.

Funding: Development of these guidelines was wholly funded by an Alternate Funding Plan Innovation grant from the Northern Ontario Academic Medical Association, a nonprofit academic medical association. The guideline was developed independent of the funding organization.

Acknowledgement: The authors acknowledge support from lan Lane of the Health Science North Research Institute for his aid in planning and execution of the in-person guideline meeting.

Endorsements: Canadian Association of Emergency Physicians, Canadian Society of Cardiac Surgery, Canadian Society of Vascular Surgery, Canadian Society of Thoracic Radiology

Correspondence to: Robert Ohle, rohle@nosm.ca 